\title{
CONTROLLING LIGHT AT THE NANOSCALE
}

I Martin Frimmer and Lukas Novotny - DOI: 10.1051/epn/2015504

v Photonics Laboratory - ETH Zürich - 8093 Zürich, Switzerland

Nanophotonics aims to control light on length scales smaller than the wavelength. By harnessing the interaction of light with matter, nanophotonics has allowed to mold the flow of light and control its emission and absorption on a length scale of just a few nanometers. 
४ P.27: Optical near-field on a laser-irradiated monocrystalline gold triangle. The image has been recorded with scanning near-field microscopy and illustrates the electromagnetic near-fields forming standing waves on the metal surface.

Image credit: Bradley Deutsch.
(1) $\mathrm{n}$ optics, lenses and mirrors are used to redirect the wavefronts of light. Due to diffraction, propagating radiation cannot be localized to dimensions much smaller than the wavelength. However, by including non-propagating fields, better localization is achievable. Nanophotonics is the science of controlling the interaction of light and matter on length scales smaller than the light's wavelength [1]. This vibrant field has created artificial materials molding the flow of light in previously unknown ways, allowed spectroscopy at the nanometer level and created light sources emitting single photons at a high rate. Instead of a comprehensive review [2], this article presents both the challenge and the promise of nanophotonics. We introduce the optical antenna as the paradigmatic tool of nano-optics and provide two illustrative examples.

\section{The challenge and the promise of sub-wavelength optics}

The challenge of manipulating radiation on length scales smaller than the wavelength arises directly from Maxwell's equations. They predict the existence of electromagnetic waves travelling in vacuum at the characteristic speed of light. A principal concept of wave physics is interference, which allows focusing of travelling waves [2]. Importantly, the smallest focus which can be generated with propagating waves of wavelength $\lambda$ is limited to about $\lambda / 2$. This limit is called the Abbe diffraction limit and for visible light is around 200 $\mathrm{nm}$. Accordingly, sub-wavelength optics is referred to as nanophotonics.

The desire to control electromagnetic fields at the sub-wavelength scale arises from the fact that light is both an ideal carrier for information as well as an extremely powerful probe. Spectroscopists harness the interaction of light with matter to gain insight into matter's properties. The building blocks of matter are atoms and molecules and spectroscopy provides insight into their internal structure and dynamics.

V FIG. 1: (a) Sketch of a metallic tip irradiated by a laser beam, creating strongly localized near-fields at its apex. The inset shows a scanning electron micrograph of a pyramidal gold nanotip used as an optical antenna. (b) Sketch of a metallic nanoparticle illuminated by a laser. The polarization of the particle creates strong near-fields in its close proximity. The inset shows a scanning electron micrograph of a gold nano-particle with a diameter of $100 \mathrm{~nm}$ attached to the end of a glass tip.

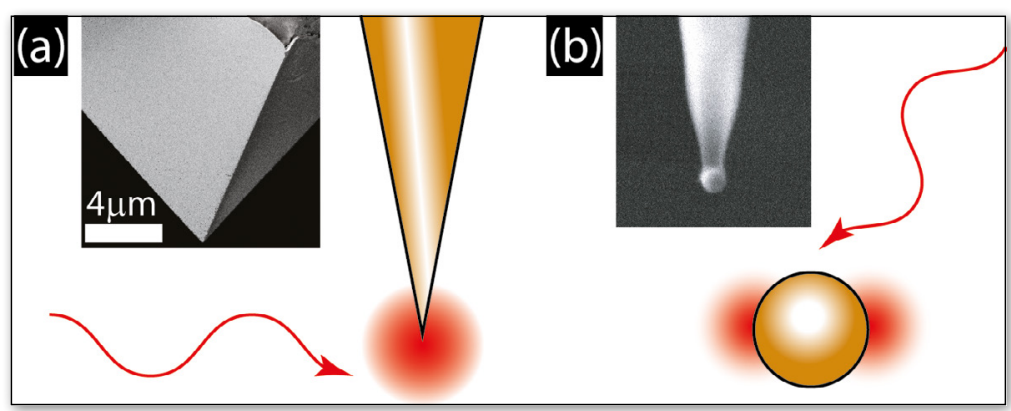

Assume that we are interested to optically probe the properties of a single molecule. Two criteria need to be satisfied. First, the interaction strength between the light field and the molecule must be large enough to detect the signature of the molecule. This interaction strength will scale with some power of the electric field strength at the molecule's position. According to Abbe's diffraction limit, the light from any source can only be focused to a volume of about $\lambda^{3}$, which poses a limit on the achievable light-matter interaction strength. Second, in order to probe a single molecule, we need to spatially resolve the molecule against all other optically responsive species in its surrounding. Accordingly, the diffraction limit imposes a limit both on the spatial resolution and the light-matter interaction strength achievable. Manipulating and focusing electromagnetic fields beyond the diffraction limit is therefore a prerequisite in order to probe matter at the single-molecule level in the crowded environments where, for example, the biochemical processes of life happen, or at the extreme integration densities of next-generation computing chips.

\section{Optical antennas}

The key to such subwavelength focusing and control is also found in Maxwell's equations. While their only physical solution in a homogeneous medium are propagating waves, at material boundaries another type of solution is allowed: the evanescent wave [1]. An evanescent wave decays exponentially away from the interface. The decay length can be much shorter than the wavelength. One method to localize evanescent waves in three dimensions is to exploit the lightning-rod effect. A lightning rod is a conductive rod with a sharp tip. When the rod is charged, strong fields are generated at its apex. Similarly, when shrunk to the nanoscale, a sharp metallic tip irradiated by a laser beam creates strong localized near-fields at its very apex, as illustrated in Fig. 1(a). In essence, such a tip acts as an optical antenna [3]. The antenna is a concept well known from electrical engineering. Antennas are devices for radiating or receiving radio waves. By extending the definition of a radio-antenna to the optical frequency regime, optical antennas mediate the emission of light from a local source or the detection of light by a local sink. Optical antennas therefore boost light-matter interaction by bridging the mismatch in length scales between the size of the emitter or receiver (about $1 \mathrm{~nm}$ for a molecule) and that of the propagating radiation set by its wavelength (which is of the order of $500 \mathrm{~nm}$ for visible light).

The lightning rod is not the only device from electrical engineering which has inspired the nanophotonics community. Particles of diameter small compared to the wavelength and irradiated with a plane wave get polarized and thereby scatter the incoming fields. In close 
proximity of the particle, these scattered fields are dominated by evanescent waves, which create hot-spots of strong light intensity in close proximity of the particle, as illustrated in Fig. 1(b). One can boost the amplitude of these evanescent fields by using a noble-metal nanoparticle which supports plasmons, collective excitations of conduction electrons. Excited at its plasmonic resonance with a driving field, such a noble-metal nanoparticle can effectively focus radiation to a volume smaller than the diffraction limit.

Just like their radiofrequency counterparts, optical antennas not only focus incoming radiation to small volumes, but equally serve as transmitting devices, boosting the power radiated by an otherwise inefficient oscillator of sub-wavelength size [4]. In the following, we present examples illustrating both sharp tips and nanoparticles acting as optical antennas.

\section{Tip-enhanced Raman spectroscopy}

One of the most impressive examples of nanophotonics enabling spectroscopy at the nanometer scale is tip-enhanced Raman spectroscopy (TERS) [6]. In Raman spectroscopy, a laser beam is sent at a material where it creates phonons, vibrational excitations of the atomic lattice. The scattered photon is shifted in energy relative to the excitation light by the phonon energy. As illustrated in Fig. 2(a), in TERS a sharp metallic tip illuminated by a laser beam is raster-scanned across a surface [5]. With the hot-spot of the electromagnetic field at the tip end being only a few $\mathrm{nm}^{3}$ in size, via the spectral content of the inelastically scattered Raman signal, a superresolution image of the vibrational properties of the species on the surface is acquired.

A particularly illustrative application of TERS is the characterization of two-dimensional materials like graphene. The superior spatial resolution provided by TERS allows to map defects and local strain in such materials, a feat not achievable with conventional farfield techniques. Figure 2(b) shows a false-color topography plot of a graphene flake (bright region) acquired by scanning a sharp metallic tip at a constant distance across the surface. At the same time as the topographical information is acquired, the Raman scattered light is collected and spectrally analyzed. Figure 2(c) shows an intensity plot of Raman spectra for tip positions along the black line in Fig. 2(b). Three different bands are clearly resolvable. The bands termed G and G' are present in the flake. These bands are the signatures of vibrational modes of the pristine graphene flake. Interestingly, there is another signature, called the D-band, generated by defects in the graphene lattice, which only shows at the very edge of the flake, which represents a defect. To illustrate the resolution provided by the technique, Fig. 2(d) shows the D-band signal as a function of position. Clearly, the D-band signal is strongly

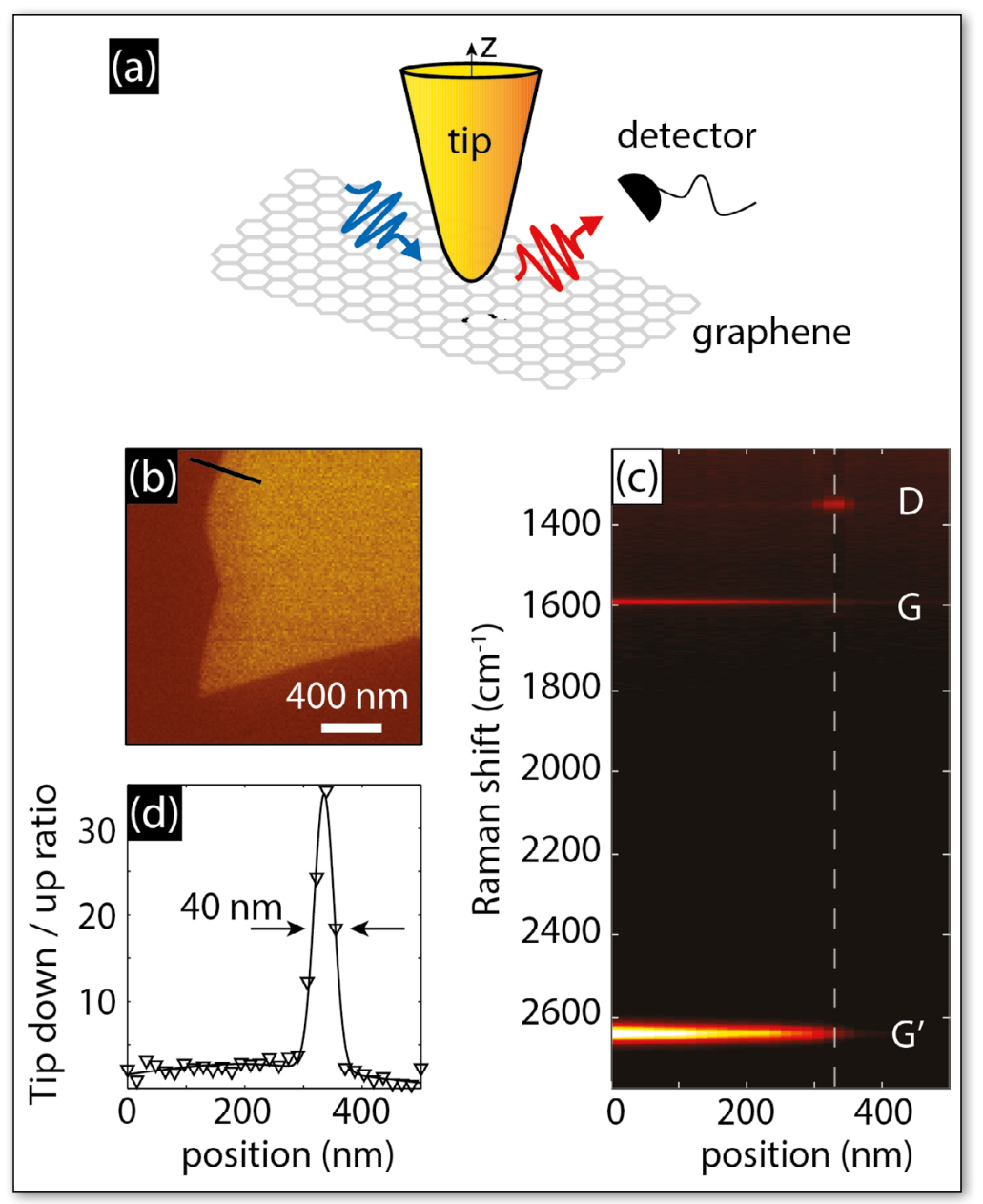

$\triangle$ FIG. 2: (a) Schematic of a TERS experiment with a graphene layer as a sample. The tip effectively focuses the excitation light (sketched in blue) at its apex. In an inelastic scattering process (red), photons are generated at characteristic Raman sidebands relative to the excitation wavelength. (b) Topography scan of graphene flake. (c) False-color plot of the Raman spectrum as a function of position on the graphene flake. The $\mathrm{G}$ and $\mathrm{G}$ ' bands are characteristic for graphene and are present in the center of the flake while at the flake edge, the D-band appears. (d) Raman-signal enhancement in the D-band due to presence of the tip as a function of position along black line in (b). The D-band signal is localized to within $40 \mathrm{~nm}$ around the flake's edge. Figure adapted from Ref. [5].

localized to within $40 \mathrm{~nm}$ around the flake's edge, while it is absent in the center of the flake, which accordingly is defect free. This measurement clearly illustrates the ability of TERS to characterize material properties with a spatial resolution not achievable with conventional microscopy techniques.

\section{Spontaneous-emission control}

A striking example of the power of optical antennas is their application to control spontaneous emission. A molecule can be promoted to an excited state via the

\section{Nanophotonics is the science of controlling the interaction of light and matter on length scales smaller than the light's wavelength $n$,}




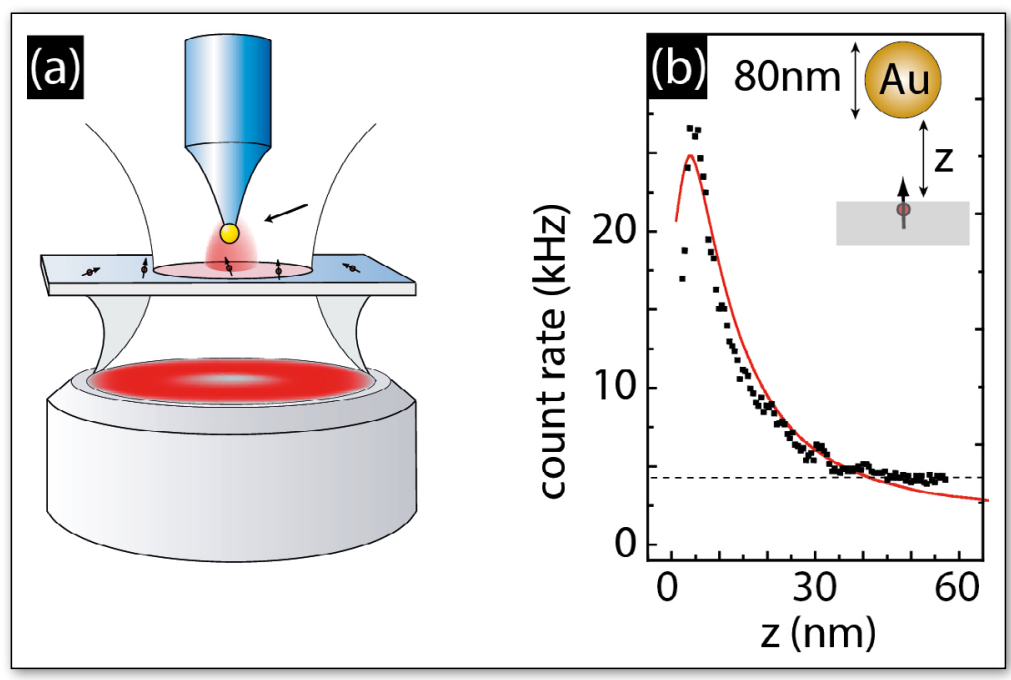

$\triangle$ FIG. 3: (a) Schematic of experiment demonstrating the fluorescence enhancement provided by an optical antenna. The antenna is a gold nanoparticle with a diameter of $80 \mathrm{~nm}$ attached to the apex of a sharp glass tip, as shown in the inset of Fig. 1(b). This tip is brought in contact with a sample on an optical microscope holding fluorescent molecules dilute enough, such that a single molecule can be addressed. (b) Fluorescence enhancement of a single molecule as a function of its distance to the nanoantenna. $0 \mathrm{n}$ a length scale of about $20 \mathrm{~nm}$ the photon detection rate is boosted by a factor of about eight by the optical antenna. Figure adapted from Ref. [8].

absorption of a photon. After a certain typical time, termed the fluorescence lifetime, the excited molecule returns to its ground state under the emission of a photon. Interestingly, this fluorescence lifetime is not solely determined by the quantum mechanical properties of the molecule. Instead, the environment determines the number of channels available for the photon generated when the molecule transitions from its excited state to the ground state. Therefore, by tailoring the environment in which a quantum emitter is located, it is possible to control its decay rate. This fact

\section{4}

\section{By extending the definition of a radio-antenna to the optical frequency regime, optical antennas mediate the emission of light from a local source or the detection of light by a local sink 79}

was first realized by E. M. Purcell in 1946 [7]. With the decay rate limiting the maximum number of photons available from a single emitter per unit time, such decay-rate engineering is of paramount importance to enhance the efficiency of light-emitting devices and to build the next-generation light sources for secure and high-speed optical data transmission. Figure 3(a) illustrates an experiment demonstrating the working principle of a simple optical antenna, a gold nanoparticle with a diameter of $80 \mathrm{~nm}$ [8]. Such a particle is attached to a glass tip, which can be positioned with nanometric precision above a sample surface. The sample holds fluorescing molecules dilute enough, such that only a single molecule is optically excited. Figure 3(b) shows a measurement of the fluorescence intensity recorded as a function of distance between the nanoparticle and the molecule. Upon approaching the optical antenna to the molecule, the detected intensity is boosted by almost a factor eight within a distance of merely $20 \mathrm{~nm}$. The observed brightness enhancement relies on two effects. First, the optical antenna effectively focuses the excitation light in its close proximity. Accordingly, the molecule is excited more efficiently by the pump light. Second, the optical antenna reduces the lifetime and therefore boosts the photon emission rate of the molecule. This experiment is a striking example of controlling the brightness of a fluorescing molecule by simply changing its relative location within its photonic environment by a subwavelength distance.

\section{Conclusion and outlook}

Nanophotonics provides a powerful toolset for spectroscopists and microscopists from the material and biological sciences. A current focus at the field's forefront is the study of light-matter interaction at the atomic and sub-atomic length scales. A major challenge for nanophotonics beyond the purely scientific playground will be to meet its promises when it comes to real-world mass applications like light management for optical computing, the realization of cheap but efficient solar cells and optical sensing.

\section{About the authors}

Lukas Novotny is a professor at the Photonics Laboratory at ETH Zürich.

Martin Frimmer is a post-doctoral researcher at the Photonics Laboratory at ETH Zürich.

\section{References}

[1] L. Novotny and B. Hecht, Principles of Nano-Optics, $2^{\text {nd }}$ Ed. (Cambridge University Press, Cambridge, 2012).

[2] A. F. Koenderink, A. Alù, and A. Polman, Science 348, 516 (2015).

[3] L. Novotny, Physics Today July, 47 (2011).

[4] T. Kalkbrenner, U. Håkanson, A. Schädle, S. Burger, C. Henkel, and V. Sandoghdar, Phys. Rev. Lett. 95, 200801 (2005).

[5] R. Beams, L. G. Cançado, S.-H. Oh, A. Jorio, and L. Novotny, Phys. Rev. Lett. 113, 186101 (2014).

[6] R. Stöckle, Y. D. Suh, V. Deckert, and R. Zenobi, Chem. Phys. Lett. 318, 131 (2000).

[7] E. M. Purcell, Phys. Rev. 69, 681 (1946).

[8] P. Anger, P. Bharadwaj, and L. Novotny, Phys. Rev. Lett. 96 , 113002 (2006). 